\title{
Efficient Multiplierless Architecture for Frame Synchronization in DVB-S2 Standard
}

\author{
Emmanuel Boutillon ${ }^{\perp}$, Pansoo Kim* ${ }^{*}$, Christian Roland ${ }^{\perp}$ and Deock-Gil Oh* \\ ${ }^{\perp}$ Université Européenne de Bretagne, UBS, Lab-STICC CNRS \\ 56100 Lorient, FRANCE \\ E-mail: emmanuel.boutillon@univ-ubs.fr, christian.roland@univ-ubs.fr \\ *Satellite Convergence Research Team, ETRI \\ 161 Gajeong-dong, Yuseong-gu, Deajeon 305-350, Republic of Korea \\ E-mail: pskim@etri.re.kr, dgoh@etri.re.kr
}

\begin{abstract}
The most challenging step of the demodulation of the DVB-S2 signal with function of VCM (Variable Coding and Modulation)/ACM (Adaptive Coding and Modulation) is the detection of the PL (Physical Layer) header. PL header is transmitted using $\pi / 2$-BPSK modulation and is composed of a fixed part (26 bits of Start Of Frame (SOF)) and a variable part (64 bits codeword of PL Signaling (PLS) code that defines the structure of the PL frame). Since the 90 bits corresponding to the PL header are affected by noise, the carrier frequency offset and the phase noise, the synchronization task in a DVB-S2 receiver is thus a critical task. In this paper, we present a properties of the Hadamard code used to encode the information of the PLS code to reinforce frame detection before knowing the actual value of the MODCOD/TYPE. Moreover, we propose to perform the computation in the polar domain in order to avoid the need of multiplier and thus, to obtain a very low cost implementation. The associated decoder architecture is presented together with the measured performance at the worst case SNR (i.e $-3 \mathrm{~dB}$ ).
\end{abstract}

\section{INTRODUCTION}

The first step of the demodulation of the DVB-S2 signal with function of VCM (Variable Coding and Modulation)/ACM (Adaptive Coding and Modulation) is the detection of the PL (Physical Layer) header in order to start the decoding process. This task is required for the determination of the starting of the frame, to overcome carrier frequency offset (all DVB-S2 transmission mode), and also to define the structure of the frame in the VCM/ACM mode [1]. In this paper, we will address only the problem of determination of the starting of the frame.

To describe the suggested detector for frame synchronization, we consider the transmission signal model of DVB-S2 standard. The received signal $r(t)$ consists of the summation of the Tx signal $s(t)$ modulated with a Binary Phase Shift Keying modulation (BPSK), i.e. $\mathrm{B}(0)=-1$ and $\mathrm{B}(1)=1$ and an Additive White Gaussian Noise (AWGN) $n(t)$ with a twosided power spectral density, $N_{o}$. Assuming that automatic gain control and symbol timing recovery circuits have been worked properly, the $\mathrm{Rx}$ signal $r(k)$ can be written as:

$$
r(k)=B(s(k)) e^{j \theta(k)}+n(k)=s(k) e^{j(\omega k+\phi(k))}+n(k)
$$

where $k$ is the time index of optimum digital sampling point, $\theta(k)=\omega k+\phi(k)$ the phase of the input symbol, $\omega$ is equal to $2 \pi \Delta f \times T_{s}$, where $\Delta f$ is the uncertainty frequency offset, $T_{s}$ is the symbol period, and $\phi(k)$ is the time-variant phase noise. If a frame starts at time $k_{0}$, then, the next 26 transmitted samples are: $s\left(k_{0}+l\right)=\operatorname{sof}(l), l=0,1, \ldots, 25$ where sof is the fixed Start Of Frame (SOF) sequence defined by the DVB$\mathrm{S} 2$ standard. The next 64 bits are $s\left(k_{0}+26+l\right)=p l s c(l) \oplus$ $\operatorname{scr}(l), l=0,1, \ldots, 63$, where, $\oplus$ is the binary Ex-Or operation, $\operatorname{scr}(l)_{l=0,1, \ldots, 63}$ is the fixed scrambling sequence defined by the standard and $p l s c(l)_{l=0,1, \ldots 63}$ is the PLS code. The PLS code $p l s c$ is defined as the product of a vector of 7 bits on information $p l s(q)_{q=0,1, \ldots, 6}$ that encode the PL frame structure (4 types of modulation, 8 code rates, $16 \mathrm{~K}$ or $64 \mathrm{~K}$ length frame size, pilot symbols or not) and the $7 \times 64$ Hadamard matrix $H$ defined as: $H(i, l)=(l>>i) \& 1$ for the first 6 lines, and $H(6, l)=1$ for the last line (i.e. the first line contains a periodic sequence of " 01 ", the second line, a periodic sequence of "0011", the third line, a periodic sequence of "00001111" and so on up to the last line that is only composed of " 1 ") $)^{1}$.

In a mass market for low cost DVB-S2 receiver, the uncertainty on the carrier frequency offset can be rather influential, leading to a value of $\omega$ between $-\pi / 2.5$ and $+\pi / 2.5$, i.e., during the reception of the 90 symbols of a PL header, up to a maximum of \pm 18 rotations of input signal can be observed $^{2}$. Moreover, the system should be able to obtain the synchronization at very low SNR (up to Es/N0 = -2.35 dB).

In the state of the art, most of papers use only the known SOF information to detect the beginning of a new frame using the combination of differential detection of symbols in order to mitigate the effect of the frequency offset. Once the frame synchronization is performed, the frequency offset estimation is done before the coherent decoding of the PLS code. In this paper, we propose to use the composition of the PLS code to perform the synchronization, using both SOF and PLS information. Moreover, in order to reduce the complexity of the synchronization process (and thus, its power dissipation), the magnitude of the input symbols $r(k)$ is first set to 1, i.e., $r(k)$ is replaced by $\arg (r(k))$ thanks to a CORDIC compo-

\footnotetext{
${ }^{1}$ The structure of the code given by the DVB-S2 standard is slightly different but leads to the same structure

${ }^{2}$ Normalized frequency offset $20 \%$ versus symbol rate
} 
nent (COordinate Rotation DIgital Computer [2]). Algorithm, associated architecture and simulated performance are given and compared to the state of the art.

The remaining part of this paper is organized as follows. Section II gives a brief state of the art on DVB-S2 synchronization issue. Section III presents the algorithm for the PLS detection. Section IV presents a joint SOF and PLS detection, and compares the performance of the new scheme to already published solution. Section V presents the architecture. Finally, conclusions are given in Section VI.

\section{State Of The ART}

In this section, we will first defined the metric to measure performance of frame synchronization. Then, we will give a rapid survey on published solutions of DVB-S2 synchronization.

\section{A. Performance evaluation of a synchronization module}

The synchronization task is a detection task, i.e., determine in the stream of incoming symbol the actual start of frame. The detector compute a metric to evaluate the likelihood that the current symbol corresponds to a start of frame or not. The output metric is then compare to a threshold to make the decision (synchronization, or not). The detector can make two types of error: first, to detect a false synchronization (false alarm), second, to miss a true start of frame (miss detection). Each value of the threshold corresponds to a couple $\left(P_{m d}, P_{f a}\right)$, where $P_{m d}$ is the probability of miss detection and $P_{f a}$ the probability of false alarm. The Receiver Operating Characteristic (ROC) curve is a 2-dimensional plot used to represent the trade-off between $P_{m d}$ (x-axis) and $P_{f a}$ (yaxis) according to the value of the threshold. Comparing the performance between different detectors can be simply done using the ROC curve. For a given false alarm rate, the one leading to the lowest miss detection rate is the most efficient. One should note that, in the DVB-S2 case, the false alarm rate should be very low. In fact, with a QPSK modulation, with long frame and no pilot, a start of frame occurs every 32,290 symbols. A false alarm probability greater than $10^{-5}$ leads to too many dummy starting of synchronization/decoding processes.

\section{B. Review of existing detectors}

In the absent of frequency offset, the optimal detector using the SOF sequence is simply a coherent correlator performing: $c(k)=\sum_{l=0}^{25} r(k-l) B(\operatorname{sof}(l))$, a normalization by the average energy of the received signal and a comparison with the threshold. With a frequency offset, is is no more possible to add coherently the received samples. Non-coherent correlator are thus used to overcome the phase rotated signal. All correlator are based on the fact that, when synchronized, $r(k-l) r(k-l-i)=B(\operatorname{sof}(l)) B(\operatorname{sof}(l+i)) e^{j \omega i}$, i.e., they can be coherently summed after suppression the contribution of $B(\operatorname{sof}(l)) B(\operatorname{sof}(l-i))$, leading to $m_{i}(k)=$ $\sum_{l=0}^{l-i} r(k-l) r(k-l-i) B(\operatorname{sof}(l)) B(\operatorname{sof}(l+i))$. The state of the art method are variations on the number of value $i$ used, the relative weighting of each value $m_{i}$ and the way the normalization factor is computed before taking the decision (see Sun et al. [3], Choi and Lee [4], Kim et al [5], [6], [7]). Especially, in case $i$ equals to 1, DPDI(Differential Post Detection Integration)is denoted. If $i$ has the maximum value within SOF symbols, it becomes D-GPDI(DifferentialGeneralized Post Detection Integration). If D-GPDI may have additional coherent and non-coherent detection component as the type of linear combination, it's called as GPDI(Generalized Post Detection Integration). CLD has D-GPDI and the energy correction term that removes possible biases in the decision variable[4]. The reference[7] allows performance close to the Maximum Likelihood (ML) detector depending channel condition given in [4] with a lower implementation cost. Nevertheless, the complexity is still $26 \times 25 / 2=325$ complex multiplications per input symbol, uniquely for the computation of the $m_{i}(k)$ values for $l=1 . .25$ [7], which remains still complex for a low cost implementation.

We should also note that, when the length of the frame is known (VCM mode) some authors propose to accumulate the $m_{i}(k)$ values over several SOF to increased the SNR. Moreover, the DVB-S2 standard suggests also to compute partial first order moment (with $i=1$ only) also on the PLS structure. In the following section, we will show how to exploit more the PLS structure to help the synchronization task.

\section{Property of the HADAMARd CODE}

In this section, the properties of the Hadamard code allowing robust PLS detection in presence of frequency offset is presented. A toy example is first presented before the generalization to the DVB-S2 Hadamard code.

\section{A. Toy example}

Let us consider a sorter Hadamard code of size $(4,8)$ for explaining the principle of the PLS detector. The encoding process associates a codeword $Y$ of size 8 to a binary vector $X$ of size 4 using:

$$
Y=X\left[\begin{array}{llllllll}
0 & 1 & 0 & 1 & 0 & 1 & 0 & 1 \\
0 & 0 & 1 & 1 & 0 & 0 & 1 & 1 \\
0 & 0 & 0 & 0 & 1 & 1 & 1 & 1 \\
1 & 1 & 1 & 1 & 1 & 1 & 1 & 1
\end{array}\right]
$$

For example, $X=[0111]$ gives the codeword $Y=$ [11000011].

The codeword $Y$ is modulated thanks to a BPSK modulation (strictly speaking, a $\pi / 2$ BPSK modulation in the DVB-S2 system): a bit zero is associated to -1 and a bit 1 is associated to 1 . Assuming a frequency offset of $\omega$ radian per sample, the received signal $R=\left(r_{k}\right)_{k=0 . .7}$ is then equivalent to:

$$
\left[1, e^{j \omega}, e^{j(2 \omega+\pi)}, e^{j(3 \omega+\pi)}, e^{j(4 \omega+\pi)}, e^{j(5 \omega+\pi)}, e^{j 6 \omega}, e^{j 7 \omega}\right]
$$

Here, we don't include the phase noise, $\phi(k)$ and noise effect, $n(k)$ to illustrate the PLS detector in Eq.(2), easily. 


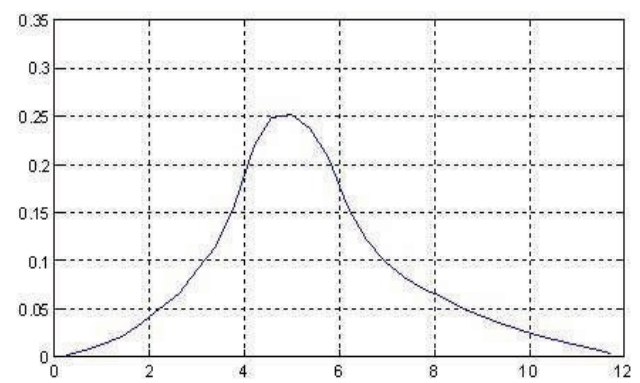

Fig. 1. Distribution of $T$ in presence of random noise

Observing this sequence, it can be noted that:

$$
\begin{aligned}
& m_{1}=r_{0} r_{1}^{*}+r_{2} r_{3}^{*}+r_{4} r_{5}^{*}+r_{6} r_{7}^{*}=4 e^{-j(\omega+0)} \\
& m_{2}=r_{0} r_{2}^{*}+r_{1} r_{3}^{*}+r_{4} r_{6}^{*}+r_{5} r_{7}^{*}=4 e^{-j(2 \omega+\pi)} \\
& m_{4}=r_{0} r_{4}^{*}+r_{1} r_{5}^{*}+r_{2} r_{6}^{*}+r_{3} r_{7}^{*}=4 e^{-j(4 \omega+\pi)}
\end{aligned}
$$

where $r^{*}$ means the conjugate of $r$.

Thus, $T=\sum_{i=1,2,4} m_{i} m_{i}^{*}=48$

It is easy to verify that the summation $T$ is independent of the codeword $Y$ and the value of the frequency offset $\omega$. Indeed, this property is inherent to the structure of the coding matrix. In the case where $R$ is composed with eight independent random values of module one, the distribution of $T$ is given in the following figure (Fig. 1 obtained by simulation over 100,000 samples).

In other words, it is clear that the value of $T$ can help to discriminate the case where the vector $Y$ corresponds to a PLS code and the case where $Y$ is just a random data. Since the PLS code of the DVB-S2 code has exactly the same property (there is just an increase in the size of the problem), we propose to use this criterion to detect the presence of a start of frame.

\section{B. PLS detector}

A generalization of the example given in section III-A for the PLS code used is quite straightforward. The only difference is that the effect of the scrambling has to be suppressed by the appropriate choice of the coefficient of the filters. The output $m_{i}(k)$ at time $k$ of the FIR filter of index $i$ is thus given by:

$$
m_{i}(k)=\sum_{j=0}^{63-i} r(k-j) r(k-j-i)^{*} h_{i}(j)
$$

The coefficients $h_{i}(j)_{j=0,1 \ldots .64-i}$ of the Finite Impulse Response (FIR) filter $H_{i}$ are given by:

$$
h_{i}(j)=B(\operatorname{scr}(j)) B(\operatorname{scr}(j+i))\left(1-\operatorname{bin}(j)\left(\log _{2}(i)\right)\right)
$$

where $B(\operatorname{scr}(\cdot))$ is the BPSK modulated scrambling sequence $(-1$ if $\operatorname{scr}(\cdot)=0,1$ if $\operatorname{scr}()=1$.$) and \operatorname{bin}(j)$ is the binary representation of $j$ and $\operatorname{bin}(j)(k)$ the $(k-1)$-th bits of this binary representation. Thus, the FIR coefficients can take the values $0,-1$ or 1 , leading to a very low implementation cost.
The output $T(k)$ is then given by:

$$
\begin{array}{r}
T(k)=\left|m_{1}(k)\right|^{2}+\left|m_{2}(k)\right|^{2}+\left|m_{4}(k)\right|^{2} \\
+\left|m_{8}(k)\right|^{2}+\left|m_{16}(k)\right|^{2}+\left|m_{32}(k)\right|^{2}
\end{array}
$$

If $T(k)$ is directly compared to a threshold $T_{P L S}$ to decide "PLS starts" or not, the ROC performance are very weak (for a given false alarm rate, the probability of detection is very low). In fact, the value of $T(k)$ is also depending with the average energy of received symbols $r(k-j)_{j=0 . .63}$. A method for improving the performance of the receiver is to mitigate the effect of module variation by a scaling factor, for example, replacing the computation of $m_{i}, i=1,2,4,8,16,32$ by the computation of $m_{i}^{\prime}$ given by:

$$
m_{i}^{\prime}(k)=\frac{\sum_{l=0}^{63-i} r(k-l) r(k-l-i)^{*} h_{i}(l)}{\sum_{l=0}^{63-i}\left|r(k-l) r(k-l-i)^{*}\right| h_{i}(l)}
$$

This operation increases the performance of the ROC curve of the receiver but still, it is rather complex to implement, since $6 \times 32=192$ complex multiplications, modules and additions, 6 divisions are required for each received sample.

To go further, we propose to simplify the complexity of the PLS detector using the phase information of the received sample $r(k) /|r(k)|$ instead of $r(k)$ itself. The first step consists to compute the phase of the received samples using a CORDIC operator.

$$
\theta(k)=\arg (r(k))
$$

Thus, using $e^{j \theta(k)}$ instead of $r(k)$, Eq. (6) is replaced by:

$$
m_{i}(k)=\sum_{l=0}^{63-i} e^{j(\theta(k-l)-\theta(k-l-i))} h_{i}(l)
$$

With this method, there is no need of normalization since norm 1 vector are used in the computation. The computation of $T(k)$ is not affected and remains identical. In the next section, we give the result of the proposed detector.

\section{Performance of the PLS detector}

In this section, we give some performance results of the PLS detector for values of $E_{s} / N_{0}$ equals to $-3 \mathrm{~dB}$ (the DVB$\mathrm{S} 2$ limit is $-2.35 \mathrm{~dB}), 0 \mathrm{~dB}$ and $3 \mathrm{~dB}$ of SNR according to the threshold $T_{P L S}$. The modulation is a QPSK modulation with long frame (64K bits, i.e., $32 \mathrm{~K}$ QPSK symbols). The probability of false alarm is defined as the average number of false alarm during the reception of a frame. As an example, a false alarm rate of $200 \%$ means that, on the average, there is two false alarms per received frame (this corresponds to a false alarm rate at a symbol level of $1 / 32400 \approx 3 \times 10^{-5}$ ). The performance given in table I are obtained using the simulation of 10000 DVB-S2 64K frames.

Using a value of $T_{P L S}=750$ means that, at $-3 \mathrm{~dB}$, on average, there is nearly one false alarm per frame and a 


\begin{tabular}{|c|c|c|c|c|c|c|}
\hline & \multicolumn{2}{|c|}{ SNR $=-3 \mathrm{~dB}$} & \multicolumn{2}{c|}{ SNR $=0 \mathrm{~dB}$} & \multicolumn{2}{c|}{ SNR $=+3 \mathrm{~dB}$} \\
\hline$T_{P L S}$ & FA & MD & FA & MD & FA & MD \\
\hline \hline 700 & $239 \%$ & $48.5 \%$ & $232 \%$ & $1.0 \%$ & $235 \%$ & $0 \%$ \\
\hline 750 & $95.1 \%$ & $54.6 \%$ & $93.6 \%$ & $1.7 \%$ & $96.4 \%$ & $0 \%$ \\
\hline 800 & $42 \%$ & $60.1 \%$ & $39.8 \%$ & $2.5 \%$ & $39 \%$ & $0 \%$ \\
\hline 850 & $16.8 \%$ & $66.8 \%$ & $16.7 \%$ & $3.5 \%$ & $16.2 \%$ & $0 \%$ \\
\hline 900 & $6 \%$ & $72.5 \%$ & $7.3 \%$ & $4.5 \%$ & $6.9 \%$ & $0 \%$ \\
\hline 950 & $2.5 \%$ & $76.5 \%$ & $3.3 \%$ & $6.1 \%$ & $3.1 \%$ & $0 \%$ \\
\hline 1000 & $0.8 \%$ & $79.4 \%$ & $1.4 \%$ & $7.8 \%$ & $1.3 \%$ & $0 \%$ \\
\hline
\end{tabular}

TABLE I

FA RATE AND MisS DETECTION RATE FOR SEVERAL VALUES OF $T_{P L S}$ AND SNR (10000 FRAMES SIMULATED).

\begin{tabular}{|c|c|c|c|c|c|c|}
\hline & \multicolumn{2}{|c|}{$T_{P L S}=700$} & \multicolumn{2}{c|}{$T_{P L S}=800$} & \multicolumn{2}{c|}{$T_{P L S}=900$} \\
\hline & $\mathrm{FA}$ & $\mathrm{MD}$ & $\mathrm{FA}$ & $\mathrm{MD}$ & $\mathrm{FA}$ & $\mathrm{MD}$ \\
\hline \hline$T_{S O F}=700$ & $0.13 \%$ & $8.94 \%$ & $0 \%$ & $9.21 \%$ & $0 \%$ & $9.45 \%$ \\
\hline$T_{S O F}=650$ & $0.2 \%$ & $8.71 \%$ & $0.01 \%$ & $9.03 \%$ & $0 \%$ & $9.31 \%$ \\
\hline$T_{S O F}=600$ & $0.4 \%$ & $8.47 \%$ & $0.04 \%$ & $8.87 \%$ & $0.02 \%$ & $9.2 \%$ \\
\hline$T_{S O F}=550$ & $0.69 \%$ & $8.06 \%$ & $0.11 \%$ & $8.55 \%$ & $0.04 \%$ & $8.94 \%$ \\
\hline$T_{S O F}=500$ & $1.23 \%$ & $7.64 \%$ & $0.23 \%$ & $8.18 \%$ & $0.08 \%$ & $8.69 \%$ \\
\hline$T_{S O F}=450$ & $2.24 \%$ & $7.16 \%$ & $0.39 \%$ & $7.77 \%$ & $0.11 \%$ & $8.41 \%$ \\
\hline$T_{\text {SOF }}=400$ & $4.2 \%$ & $6.56 \%$ & $0.68 \%$ & $7.28 \%$ & $0.12 \%$ & $8.08 \%$ \\
\hline \hline
\end{tabular}

TABLE II

FA RATE AND MisS DETECTION RATE FOR JOINT SOF AND PLS DETECTION AT A SNR OF 0 DB (10000 FRAMES SIMULATED).

probability of 0.5 to have the PLS detection. If the FA case is rapidly processed, then, on average, PLS synchronization can be obtained in two frames. One should note that the performance is similar when others type of modulations are considered for the frame (8-PSK or 16-APSK).

\section{JOINT SOF/PLS DETECTOR}

In order to improve the performance of the frame detection, we have combine SOF and PLS detections. In other words, a start of frame is detected if both SOF detection and PLS detection are respectively above two chosen thresholds $T_{S O F}$ and $T_{P L S}$.

Let $R(k)$ be the input of the SOF filter at time $k^{\prime}=k-64$ (in order to be synchronized with the output of the PLS filter), thus, $R(k)$ can be written as:

$$
R(k)=\sum_{i=0}^{25}\left|n_{i}\left(k^{\prime}\right)\right|^{2}
$$

where $n_{i}\left(k^{\prime}\right)$ is given by:

$$
n_{i}\left(k^{\prime}\right)=\sum_{l=0}^{26-i} e^{j\left(\theta\left(k^{\prime}-l\right)-\theta\left(k^{\prime}-l-i\right)\right)} g_{i}(l)
$$

The coefficients $g_{i}(l)_{l=0,1 \ldots(26-i)}$ of the FIR filter $G_{i}$ are given by $g_{i}(l)=B(\operatorname{sof}(l)) B(\operatorname{sof}(l+i))$.

Table II gives the obtained performance for an SNR of 0 dB.

One should note that the addition of the SOF leads to a significant increase of the performance. In particular, it is

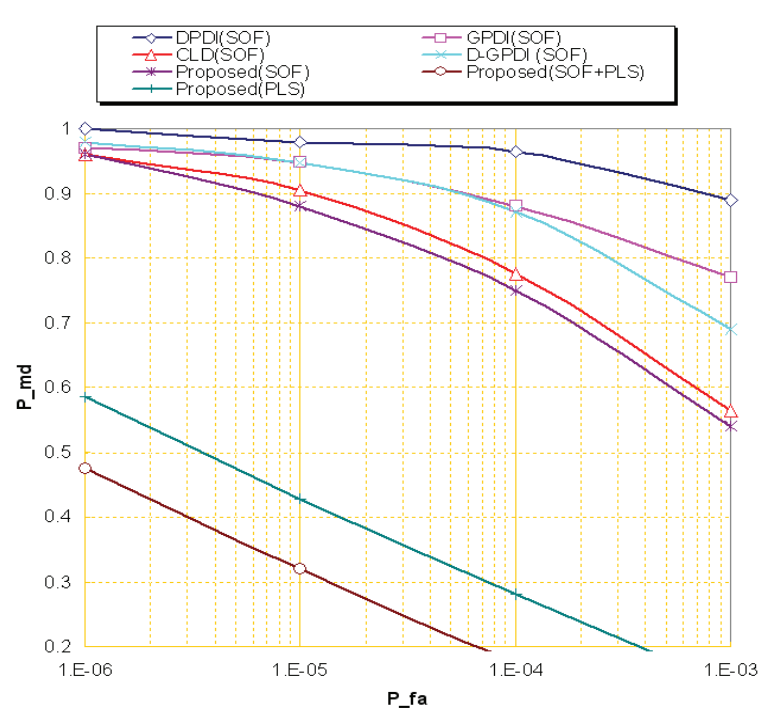

Fig. 2. ROCs for SOF detector optimization plus the SOF+PLS detector optimization

possible to reduce the rate of false alarm for a given miss detection rate.

Fig. 2 shows the performance in terms of ROCs for all competitive correlators in order to choose the most appropriate one in the single dwell process. The ROC curve is obtained for $E_{s} / N_{o}=-3 d B$ and a normalized freq. offset of $10 \%$. First, one should note that the determination of PLS approach using only SOF with phase information leads to a slightly better results than with the CLD detector, with much simpler architecture, since complex multiplications are replaced by phase subtractions. This is the first contribution of this paper. Second, combining SOF with PLS detection dramatically reduces the FA rate for a given frame Miss Detection rate. For example, for a false alarm rate of $10^{-5}$ (one false alarm per frame in average), SOF alone leads to a miss detection rate of around 0.9 and PLS alone leads to a miss detection rate of about 0.43 . When combined, this leads to a miss detection rate of 0.32 . The efficiency of joint SOF-PLS based detection method is the second main contribution of this paper.

\section{Architecture of SOF/PLS Detector}

In this section, we first present the precision issue, then the architecture and we give a rapid complexity estimation of the whole PLS detection.

\section{A. Precision issue}

In order to evaluate the required precision of the algorithm, we have quantized $\theta(k)$ values on several number of bits. The quantized value $\theta_{q}$ of $\theta$ on $n_{b i t}$ bits is given by:

$$
\theta_{q}=\left\lfloor\theta /(2 \pi) \times 2^{-n_{b i t}}+0.5\right\rfloor
$$

where $\lfloor x\rfloor$ represents the highest integer that is smaller of equal to $x$. 


\begin{tabular}{|c|c|}
\hline$n_{\text {bit }}$ & Miss detection \\
\hline 2 & 294 \\
\hline 3 & 99 \\
\hline 4 & 23 \\
\hline 5 & 14 \\
\hline 6 & 12 \\
\hline 7 & 12 \\
\hline floating point & 12 \\
\hline
\end{tabular}

TABLE III

Missed DETECTION AS A FUNCTION OF THE QUANTIZED VALUE OF $\theta(k)$

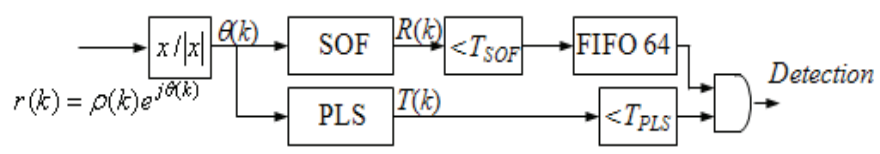

Fig. 3. Architecture of PLS/SOF detector

Table III shows the number of miss detection as a function of the number $n_{b i t}$ of bit used to quantized $\theta(k)$ for a SNR of $6 \mathrm{~dB}$ and threshold of SOF and PLS respectively equal to $T_{S O F}=700$ and $T_{P L S}=900$.

As can be seen in table III, the coding of $\theta(k)$ on 6 bits is adequate. For a given value of $\theta_{q}$, the computation of $e^{j \theta_{q}}$ is simply done by a read access in a ROM of size $2^{6}=64$ that contains both the real and the imaginary parts of the result, which is also coded on 6 bits $^{3}$.

\section{B. PLS/SOF architecture}

The whole architecture is presented on fig. 3. The first step is to determine the phase of the received signal $r(k)$ using a CORDIC component. Then, $\theta(k)$ is sent to the SOF and PLS detectors. The output of the two filters are compared to the thresholds $T_{S O F}$ and $T_{P L S}$ to generate SOF and PLS alarms. The SOF alarm is first delayed of 64 clock cycles, in order to be synchronized with the PLS alarm. If both are positive, a PLS start detection is decided.

The architecture of the PLS and SOF detection are very similar. They are given respectively in fig. 4 and fig. 5. In fig. 4, the coefficient of the Finite Impulse (FIR) filter are -1 and 1 and the size of filter $G_{i}$ is $26-i$. The quantity of memory is then 325 words of 12 bits (complex number coded on 6 bits). The computational complexity to obtain an output sample $R(k)$ is: 25 accesses in a ROM $64 \times 12$ (for phase to complex conversion), 325 complex additions, 25 square operations and 24 adders for the final summation.

In fig.5, the FIR filter have a size of $64-i$ with only 32 non-zero coefficients taking there values between -1 et 1 . The quantity of memory is 321 words of 12 bits. The computational complexity to obtain an output sample $T(k)$ is: 6 accesses in a ROM $64 \times 12$ (for phase to complex conversion), 192 complex additions, 6 square operations and 5 adders for the final summation.

According to the ratio of the clock frequency and the sample rate, it is easy to derives an efficient architecture.

${ }^{3}$ It can be further reduced using the symmetries of the cosine and sine functions.

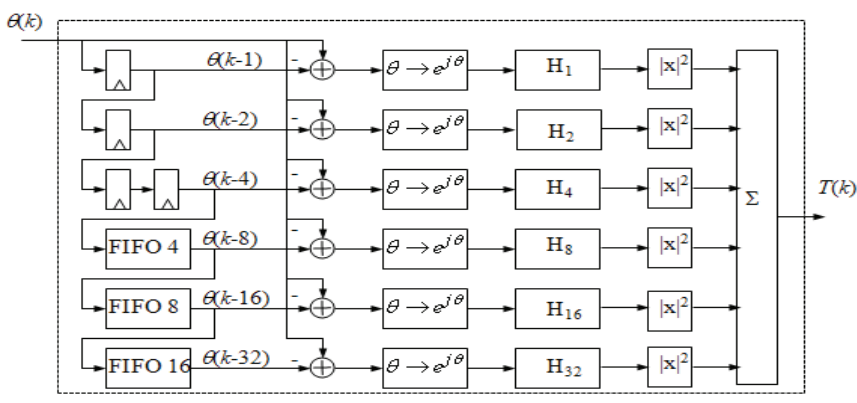

Fig. 4. Architecture of SOF detector

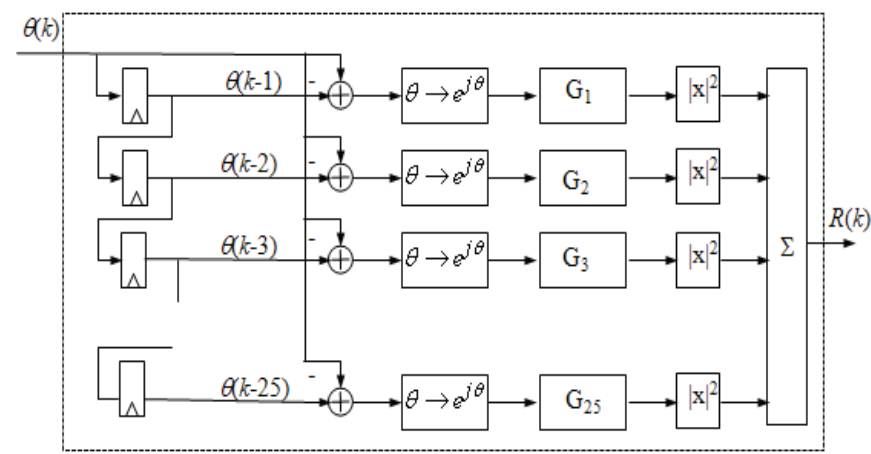

Fig. 5. Architecture of PLS detector

\section{CONCLUSIONS}

This paper proposes a robust correlator for frame synchronization in DVB-S2 receiver. The proposed one can identify the location of SOF (Start of Frame) reliably because it collects much signal energy using Hadamard generator principle in the PLSCODE. Since it doesn't have any multiplicative operation, it is possible to carry out high speed operation in case of $\mathrm{H} / \mathrm{W}$ implementation. It shows better performance in terms of ROC than those obtained with state of the art algorithms.

\section{REFERENCES}

[1] ETSI EN 302 307, v. 1.1.2 Digital Video Broadcasting (DVB); Second generation framing structure, channel coding and modulation systems for broadcasting, interactive services, news gathering and other broadband satellite applications, June 2006.

[2] J. E. Volder, "The CORDIC Trigonometric Computing Technique", IRE Transactions on Electronic Computers, Sept. 1959.

[3] F. Sun, Y. Jiang, L. Lee, "Frame synchronization and pilot structure for second generation DVB via satellites, "Wiley Int. Journal of Satellite Communications and Networking, Vol. 22, no. 3, pp.319-339, May-Jun 2004.

[4] Z. Y. Choi, and Y. H. Lee, "Frame synchronization in the presence of frequency offset," IEEE Trans. on Commun., Vol. 50, no. 7, pp.11621065, Jul. 2002.

[5] P. Kim, et al, "Enhanced frame synchronization for the DVB-S2 system under a large of frequency offset, " in Proceedings of IEEE Wireless Communications and Networking Conference (WCNC'07), , pp.11831187, Mar 2007.

[6] P. Kim, et al, "Robust frame synchronization for the DVB-S2 system with large frequency offsets, "Wiley Int. Jour. of Satellite Communications and Networking, Vol. 27, no. 1, pp.35-52, Jan-Feb 2009.

[7] D.-U. Lee, P. Kim, and W. Sung, "Robust frame synchronization for Low Signal-to-Noise Ratio Channels Using Energy-Correlated Differential Correlation, "Eurasip Journal on Wireless Communications and Networking, Vol. 2009. 